\title{
Status of the MINOS experiment
}

\author{
Milind V. Diwan ${ }^{\mathrm{a}} *$ \\ ${ }^{a}$ Brookhaven National Laboratory, \\ Upton, NY, 11973 \\ I will present the status of the long baseline neutrino oscillation experiment MINOS at Fermi National Accel- \\ erator Laboratory (Fermilab). I will summarize the status of the detector and beam construction, the expected \\ event rates and sensitivity to physics. I will also comment on possible future plans to improve the performance \\ of the experiment.
}

\section{Introduction}

The strongest evidence for neutrino oscillations comes from astrophysical observations of atmospheric neutrinos with $\Delta m_{a t m}^{2}=(1.6-4.0) \times$ $10^{-3} \mathrm{eV}^{2}$ and maximal mixing [1, 2], and from solar neutrinos with $\Delta m_{\text {solar }}^{2}=(3-10) \times 10^{-5} \mathrm{eV}^{2}$ assuming the LMA solution 3, 4, 5. The observation by the LSND experiment [6] will soon be re-tested at Fermilab by the mini-Boone [7] experiment. Therefore we will not discuss it further in this document. There is now a consensus that there are four main goals in the field of neutrino oscillations that should be addressed soon with accelerator neutrino beams:

1. Precise determination of $\Delta m_{32}^{2}=\Delta m_{\text {atm }}^{2}$ and $\sin ^{2} 2 \theta_{23}$ and definitive observation of oscillatory behavior.

2. Detection of $\nu_{\mu} \rightarrow \nu_{e}$ in the appearance mode. If the measured $\Delta m^{2}$ for this measurement is near $\Delta m_{32}^{2}$ then this appearance signal will show that $\left|U_{e 3}\right|^{2}\left(=\sin ^{2} \theta_{13}\right)$ from the neutrino mixing matrix in the standard parameterization is non-zero.

3. Detection of the matter enhancement effect in $\nu_{\mu} \rightarrow \nu_{e}$ in the appearance mode. This effect will also allow us to measure the sign of $\Delta m_{32}^{2}$, i.e. which neutrino is heavier.

\footnotetext{
*Representing the MINOS collaboration which has $\sim 200$
} collaborators from 30 institutions.
4. Detection of $\mathrm{CP}$ violation in neutrino physics. The neutrino CP-violation in the standard neutrino mixing model comes from the phase of $U_{e 3}$ in the mixing matrix. This phase causes an asymmetry in the oscillation rates $\nu_{\mu} \rightarrow \nu_{e}$ versus $\bar{\nu}_{\mu} \rightarrow \bar{\nu}_{e}$.

In this paper I will describe how the MINOS experiment [8,9, 10,11, 12] will make the first important contributions to this program of physics.

\subsection{The neutrino beam}

A new neutrino beamline, called NuMI, has been constructed at Fermilab [11]. High energy, $120 \mathrm{GeV}$, protons from the main injector with intensity of $4 \times 10^{13}$ every $1.9 \mathrm{sec}$ in $8.1 \mu$ s-long pulse will strike a $1 \mathrm{~m}$-long segmented graphite target. The secondary mesons are collected by a two-horn focusing system and directed towards the Soudan Iron Mine in Minnesota, $735 \mathrm{~km}$ away, at an angle of $58 \mathrm{mrad}$ into the ground. The mesons, most of which are charged pions, are sign selected to be positive by the horn system and allowed to decay in a $675 \mathrm{~m}$ long and $2 \mathrm{~m}$ diameter evacuated decay tunnel. The two-horn wide band system allows us to tune the mean energy of the $\nu_{\mu}$ from about 3 to $18 \mathrm{GeV}$. Since the latest oscillation parameters from the Super Kamiokande atmospheric neutrino data are in the range of $\sim 3 \times 10^{-3} \mathrm{eV}^{2}$, the first run of MINOS will most likely be with the lowest energy beam to get as large an oscillation effect as possible for the $735 \mathrm{~km}$ baseline. The expected $\nu_{\mu}$ flux from the NuMI beamline is shown in Figure 1. The contamination from 


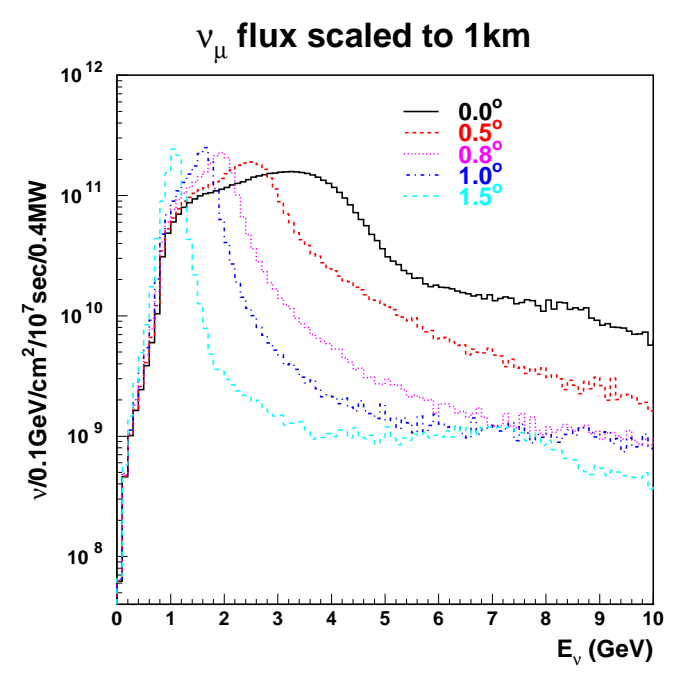

Figure 1. The muon neutrino flux expected from the NuMI beam. The flux is normalized for 0.4 MW of $120 \mathrm{GeV}$ proton for $10^{7} \mathrm{sec}$ of running time. The flux is scaled to be at $1 \mathrm{~km}$ distance from the NuMI target, but the spectrum shape was calculated for $735 \mathrm{~km}$. The spectrum expected at several angles to the $0^{\circ}$ axis is also shown.

electron neutrinos will be less than $1 \%$ with approximately the same spectrum.

\subsection{The MINOS detector}

The MINOS experiment has two detectors, the near detector and the far detector, to determine the neutrino flux close to the source as well as $735 \mathrm{~km}$ away. The near detector, with a mass of $\sim 1 \mathrm{kTon}$, will be placed $1040 \mathrm{~m}$ downstream of the target, or about $300 \mathrm{~m}$ beyond the end of the decay tunnel, and about $100 \mathrm{~m}$ underground. It is designed to precisely measure neutrino beam characteristics such as the energy spectrum and the $\nu_{e}$ contamination. The near detector is constructed to be nearly identical to the far detector in terms of segmentation so that the event reconstruction, background levels, and energy resolu-

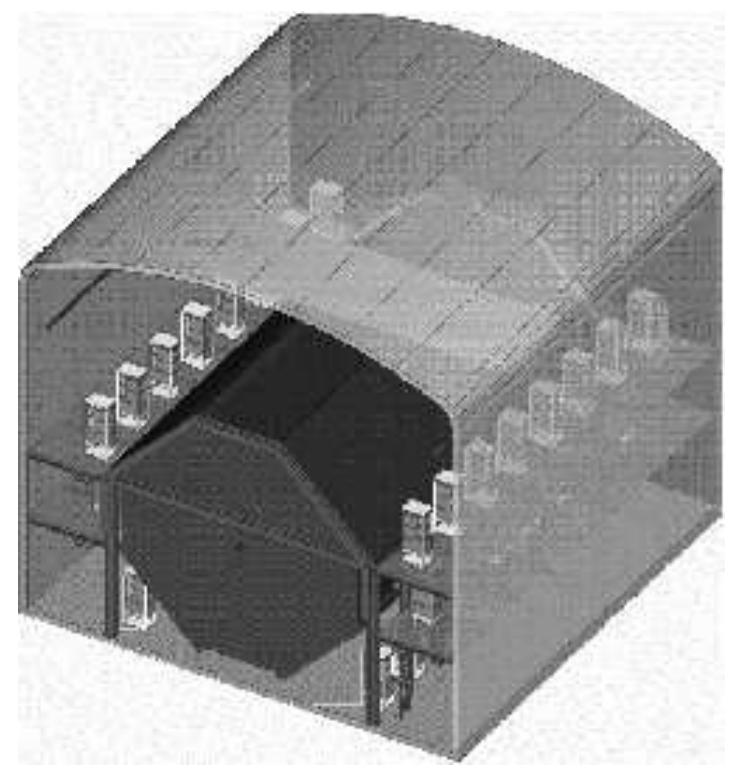

Figure 2. Schematic of the MINOS far detector. The octagonal area is the mainly iron detector mass; the central hole accomodates a coil to generate the toroidal magnetic field. The racks on both sides of the detector house the multi-anode photomultipliers and the front end electronics.

tion can be studied with the unoscillated neutrino flux with high statistics. The neutrino interaction rate in the near detector is sufficient to provide nearly continuous beam monitoring. For the final analysis we expect that interations in the central region of $25 \mathrm{~cm}$ radius will be used. Beam monitoring will also be provided by a set of ceramic pad ionization chambers placed in 3 caves after the end of the decay tunnel. These chambers were designed to provide stable, precise monitoring of the secondary muon flux as a function of time as well as azimuthal position [12].

The far detector has a total mass of $5.4 \mathrm{kT}$. It is placed in a new cavern dug $713 \mathrm{~m}$ underground in the Soudan mine in northern Minnesota, about $735 \mathrm{~km}$ away from the primary target at Fermilab. The far detector is made out of two supermodules, each an 8m-diameter octagonal toroid 
composed of 243 layers. Each layer is made of a $2.54 \mathrm{~cm}$-thick steel plane and $1 \mathrm{~cm}$-thick and $4.1 \mathrm{~cm}$-wide scintillator strips grouped in 20- or 28 -strip wide light-tight modules. The scintillator strips are made in an industrial extrusion process using Styron $663 \mathrm{~W}$ polystryrene, manufactured by the DOW chemical company, doped with $1 \%$ PPO and $0.03 \%$ POPOP. A $0.25 \mathrm{~mm}-$ thick reflective layer, made by adding $12.5 \% \mathrm{TiO}_{2}$ to polystyrene, is co-extruded with the scintillator strips. A $1.4 \mathrm{~mm}$-wide and $2 \mathrm{~mm}$-deep groove in the center of the $4.1 \mathrm{~cm}$-side is also made during the extrusion process. A $1.2 \mathrm{~mm}$ diameter wave length shifting (WLS) fiber is embedded in the groove during the assembly of the scintillator modules. The J-type Y11 multiclad PMMA, nonS WLS fiber made by Kuraray and doped with the K27 dye at $175 \mathrm{ppm}$ (with maximum intensity emission at $520 \mathrm{~nm}$ ) is used. The fibers are optically coupled to the scintillator strips with epoxy. The WLS fibers are read out from both ends. They are grouped (multiplexed) inside a lighttight box into sets of 8 fibers from strips spaced more than $1 \mathrm{~m}$ apart in each plane. Each 8-fiber bundle is coupled, using a "cookie", to a single pixel of a 16-pixel R5900-M16 Hamamatsu photomultiplier (PMT). Thus each PMT reads out 128 fibers; one end of each scintillator plane needs 24 pixels. This arrangement allows us to read out the entire MINOS far detector using only 1452 PMTs. Since the event rate is small, unambiguous event recontruction can be achieved in software despite the multiplexing. An important detector parameter is the photo-electron yield for a minimum ionizing particle (MIP) incident at right angle to the scintillator strip: the average yield, measured using a radioactive source for each strip during assembly, is about 6 photo-electrons per MIP summed from both sides. The attenuation over the 8 meter length of the strip is about a factor of 3 .

The near detector is a $3.8 \mathrm{~m}$ by $4.8 \mathrm{~m}$ "squeezed" octagonal toroid made of solid 2.54 $\mathrm{cm}$ iron plates with scintillator strips of similar design as the far detector between the iron plates. The near detector has two longitudinal sections: The first 96 planes are called the target or the hadron/shower calorimeter. In these planes the area that will be exposed to the central part of the beam (about half of the tranverse area) has scintillator modules in every plane. Every 4th plane in the hadron/shower calorimeter has full area scintillator coverage. The second, muon tracking, section has 164 planes with every 5th gap instrumented with full area scintillator coverage. The fibers are read out individually (no multiplexing) from one end only by 215 64-anode Hamamatsu PMTs. The opposite ends of the fibers are reflectively coated to increase the average light yield to similar level as the far detector.

Both detectors are magnetized using a coil through a hole in the center of the planes to an average field of about $1.5 \mathrm{~T}(2 \mathrm{~m}$ away from the coil). The front end electronics is different for the two detectors because the event rate in the $8.1 \mu$-sec neutrino pulse in the near detector is far higher than the far detector. In the far detector the read out electronics is based on a VA chip from IDE and in the near detector it is based on the QIE chip designed at Fermilab. Simulations show that these detectors have a resolution of $60 \% / \sqrt{E}$ for hadronic showers and $25 \% / \sqrt{E}$ for electromagnetic showers. Both detectors are being calibrated by cosmic rays and a light injection system. A test beam calibration module is being used to perform relative calibration between the near and far detectors of about $2 \%$ and absolute calibration of about $5 \%$.

The MINOS experiment is currently under construction. Approximately $2 / 3$ of the detector has been assembled in the Soudan mine and is taking cosmic ray data. The civil construction for the NuMI beam line is finished and the technical items are now being assembled. Data with the neutrino beam is expected in December 2004.

\section{The physics sensitivity}

A summary of the important parameters of the MINOS experiment is shown in Table 1.

\subsection{Muon neutrino disappearance}

MINOS plans to detect oscillations by observing the absolute event rate as well as the energy distribution for charged current muon neutrino events. We will also obtain a confirming oscil- 
Table 1

Parameters of the MINOS experiment

\begin{tabular}{ll}
\hline Proton Energy & $120 \mathrm{GeV}$ \\
Time Structure & $8.1 \mu \mathrm{s}$ every $1.9 \mathrm{sec}$ \\
Intensity & $4 \times 10^{13}$ protons/spill \\
Exposure & $3.8 \times 10^{20}$ protons/year \\
Beam & $1 \mathrm{~m}$ graphite target 2 -horn pion focusing \\
& LE $1-6 \mathrm{GeV} \nu_{\mu}$ with tail to $50 \mathrm{GeV}$ \\
Baseline & $735.340 \mathrm{~km}$ \\
Detector & $2.54 \mathrm{~cm}$ Iron, $1 \mathrm{~cm}$ scint. \\
& Magnetized Average field $1.5 \mathrm{~T}$ \\
Resolution & hadronic $60 \% / \sqrt{E}$ \\
& electromagnetic $25 \% / \sqrt{E}$ \\
Event rate & Near det. 3 events $/$ spill in target region \\
& Far det. $300 \nu_{\mu} \mathrm{CC}$ events $/ \mathrm{kT} / \mathrm{yr}$ (no osc) \\
\hline
\end{tabular}

lation signature in the ratio of the number of charged and neutral current events. The total neutrino energy will be measured by measuring the muon momentum and range as well as the total energy of the hadron shower. The resulting distribution of reconstructed neutrino energy is shown in Figures 3 and 1 , for $\Delta m^{2}=0.002 \mathrm{eV}^{2}$ and $\Delta m^{2}=0.0035 \mathrm{eV}^{2}$, respectively; $\sin ^{2} 2 \theta=$ 0.9 for these plots. To extract oscillation parameters it will be necessary to precisely predict the spectrum without oscillations at the far detector using the near detector data. Using known tolerances on the target/horn geometry as well as the differences in the near and far reconstrution efficiencies we expect to reduce the systematic error on the far spectrum to about $2 \%$ in normalization and $2 \%$ in bin-to-bin fluctuations. Using these systematic uncertainties we predict that MINOS will be able to measure the oscillation parameter $\Delta m^{2}$ to $\pm 10 \%$ at the best fit point of the SuperKamiokande allowed region. This is shown in Figure 5. The Super-Kamiokande best fit point is now at $0.0025 \mathrm{eV}^{2}[13]$. For this value, the minimum in the oscillation probability for MINOS will be at $1.5 \mathrm{GeV}$ which is on the lower rising edge of the charged current spectrum (See the unoscillated histogram in Figure 3). Therefore, one possibility under study for improved performance is to increase the flux below $2 \mathrm{GeV}$ by appropriate modifications to the target/horn geometry.

\subsection{Electron neutrino appearance}

The main oscillation channel in the case of atmospheric oscillations is now thought to be $\nu_{\mu} \rightarrow \nu_{\tau}$ [2]. Nevertheless, in the 3 neutrino formulation of oscillations it is natural to expect a small component of the disappearing $\nu_{\mu}$ to convert to $\nu_{e}$ with the same oscillation length as the atmospheric one. This will occur if the presently unknown neutrino mixing matrix element $U_{e 3}$ is nonzero. The best limit on $U_{e 3}$ currently comes from the reactor experiment Chooz 14 in which a search was made for disappearance of $\bar{\nu}_{e}$. At the Super Kamiokande best fit $\Delta m^{2}=0.0025 \mathrm{eV}^{2}$ the Chooz limit implies $\left|U_{e 3}\right|^{2}<0.03$ at $90 \%$ confidence level. The expected sensitivity of MINOS for $\nu_{\mu} \rightarrow \nu_{e}$ appearance is shown in Figure 6 . With $10 \mathrm{kT}$-year of exposure we expect to improve the Chooz limit by about a factor of 2 . At $\Delta m^{2}=0.0025 \mathrm{eV}^{2}$ and $\left|U_{e 3}\right|^{2}=0.03$ we expect an excess of 24 electron event over a background of 40 events. For Figure 6 we assumed that the background will be known to about $10 \%$ by using the near detector data. The sensitivity is limited by the neutral current background (28 events) out of which approximately half will be from high en- 


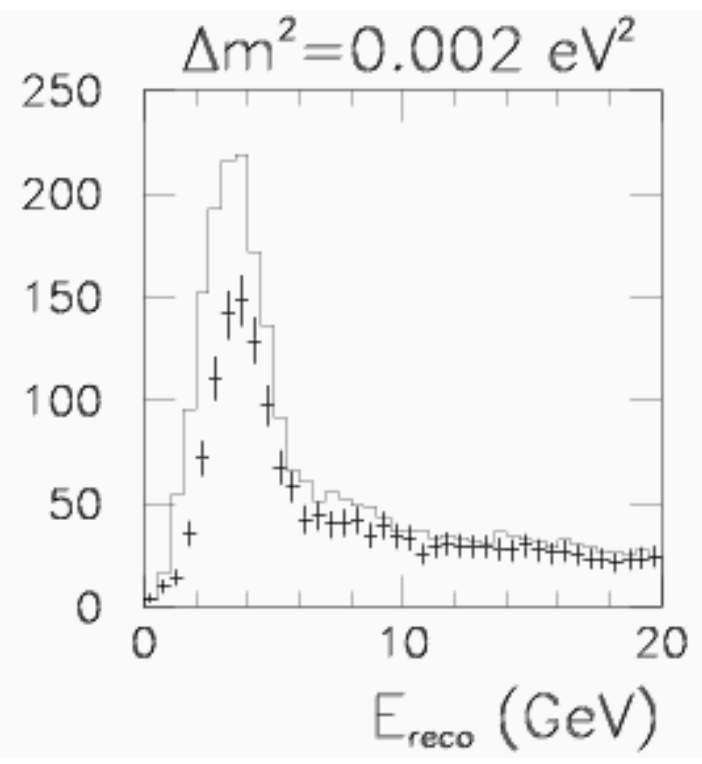

Figure 3. Expected spectrum of reconstructed muon neutrino energy for $\Delta m^{2}=0.002 \mathrm{eV}^{2}$ and $\sin ^{2} 2 \theta=0.9$. We assume 10kT-year of exposure in the low energy beam for this result.

ergy $(>10 \mathrm{GeV})$ neutrinos. We are examining the possibility of reducing the background and improving the signal by 1) eliminating the high energy tail of the beam by using a "beamplug", 2) improving the flux at low energies. A more sensitive approach to $\nu_{\mu} \rightarrow \nu_{e}$ has been proposed to use the off-axis narrow band NuMI beam (See Figure 1) 15 and a new detector placed on the Earth's surface near Soudan 16]. With an upgraded more intense main injector such an experiment could also be sensitive to CP violation. Ultimately, for the best sensitivity to matter effects and $\mathrm{CP}$ violation using a conventional neutrino beam one may have to go to much longer baselines 17, 18.

\section{Conclusion}

The NuMI neutrino beamline and the MINOS near and far detectors are being constructed. Ap-

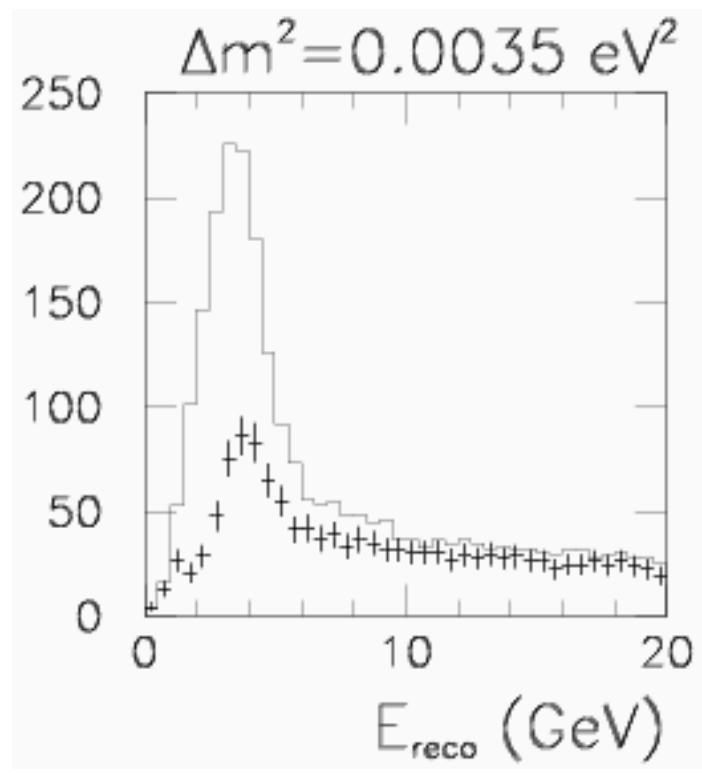

Figure 4. Expected spectrum of reconstructed muon neutrino energy for $\Delta m^{2}=0.0035 \mathrm{eV}^{2}$ and $\sin ^{2} 2 \theta=0.9$. We assume $10 \mathrm{kT}$-year of exposure in the low energy beam for this result.

proximately $2 / 3$ of the $5.4 \mathrm{kT}$ MINOS detector has been assembled in the Soudan mine; it is now gathering cosmic ray data. The NuMI beamline will be finished in 2004 and data gathering with the neutrino beam will commence in December of 2004 . With $10 \mathrm{kT}$-year of data with $3.8 \times 10^{20}$ protons per year we expect to confirm the oscillatory behaviour of muon neutrinos and measure $\Delta m^{2}$ with $10 \%$ error. We will also improve the limit on $\nu_{\mu} \rightarrow \nu_{e}$ beyond the limit of Chooz or discover this appearance channel if the parameter $\left|U_{e 3}\right|^{2}$ is sufficiently large. The sensitivity to the appearance channel is limited by the neutral current background, and we are examining various possibilities to reduce this background.

\section{REFERENCES}

1. Y. Fukuda et al., Phys. Rev. Lett. 81, 1562 (1998) 


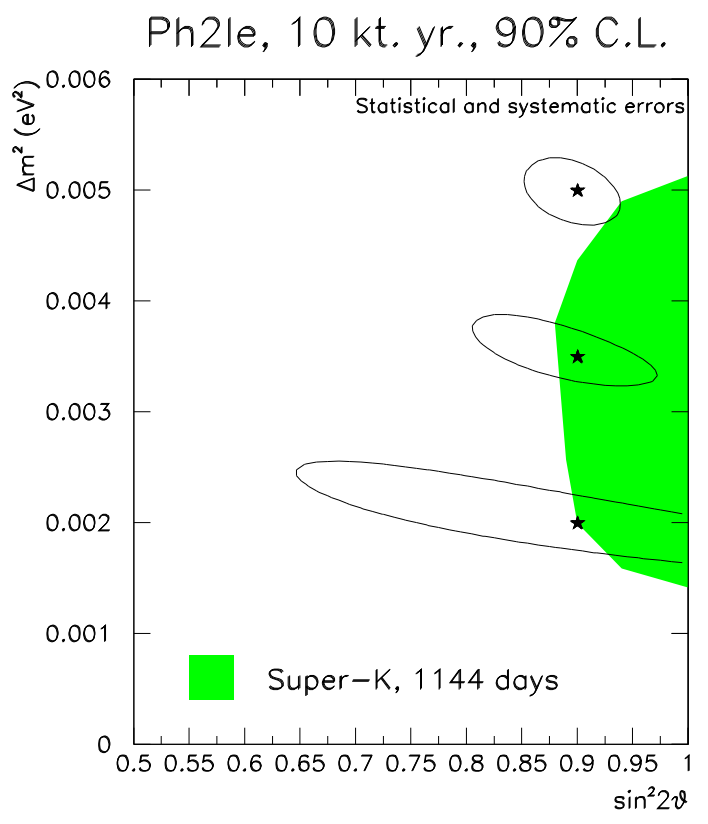

Figure 5. Expected resolution from MINOS from the disappearance signature for selected parameters (the stars). The favored region from Super Kamiokande data is also indicated.

2. S. Fukuda et al., Phys. Rev. Lett. 85, 3999 (2000)

3. Q. R. Ahmad et al., Phys. Rev. Lett. 87, 071301 (2001)

4. Q. R. Ahmad et al., Phys. Rev. Lett. 89, 011301 (2002).

5. Q. R. Ahmad et al., Phys. Rev. Lett. 89, 011302 (2002).

6. C. Athanassopoulos et al., Phys. Rev. Lett. 773082 (1996); C. Athanassopoulos et al., Phys. Rev. Lett. 811774 (1998)

7. Booster Neutrino Experiment, Fermi National Laboratory, http:/www-boone.fnal.gov/

8. Fermilab Proposal P875, February, 1995. The MINOS technical design report, Fermilab, NuMI-L-337, October, 1998.

\section{MINOS $v_{\mu} \rightarrow v_{e}$ sensitivity}

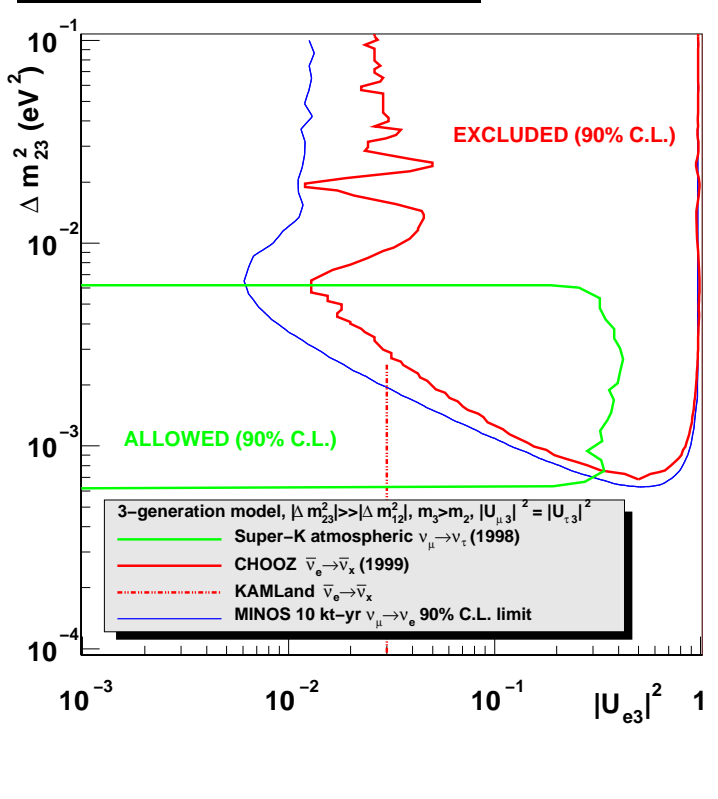

Figure 6. Expected 90\% confidence level limit for $\nu_{\mu} \rightarrow \nu_{e}$ appearance if MINOS sees no excess of electron neutrino events with $10 \mathrm{kT}$-year of exposure.

9. P. Adamson et al., Nucl. Instrum. Meth. A492 325-343 (2002)

10. K. Lang et al., Nucl. Instrum. Meth. A461 571 (2001)

11. J. Hylen et al., FERMILAB-TM-2018, Sep 1997.

12. J. Mcdonald et al., Archive:physics/0205042, May 2002, To be published in Nucl. Instrm. Meth.

13. T. Kajita and Y. Totsuka, Rev. Mod. Phys. 73, 85 (2001).

14. M. Apollonio et al., Phys. Lett., B466, 415 (1999).

15. Long Baseline Neutrino Oscillation Experiment, D. Beavis et al., Physics Design Report, BNL-52459. April 1995.

16. Letter of Intent to build an Off-axis Detector to study $\nu_{\mu} \rightarrow \nu_{e}$ oscillations with the NuMI Neutrino Beam, D. Ayres et.al, hep$\mathrm{ex} / 0210005$ 
17. William J. Marciano, arXiv: hepphy/0108181, 22 Aug 2001.

18. Report of the BNL Neutrino Working Group: Very Long Baseline Neutrino Oscillation Experiment for Precise Determination of Oscillation Parameters and Search for $\nu_{\mu} \rightarrow \nu_{e}$ Appearance and CP Violation, M. diwan, et al., BNL-69395, Oct 28, 2002. hep-ex/0211001. 